Archives de sciences sociales des religions

113 | janvier-mars 2001

Varia

\title{
Mystagogie et religions de guérison : Max Weber revisité
}

Régis Dericquebourg

\section{OpenEdition}

Édition électronique

URL : http://journals.openedition.org/assr/20196

DOI : 10.4000/assr.20196

ISSN : $1777-5825$

Éditeur

Éditions de l'EHESS

Édition imprimée

Date de publication : 1 avril 2001

Pagination : 149-158

ISBN : 2-222-96701-5

ISSN : 0335-5985

Référence électronique

Régis Dericquebourg, « Mystagogie et religions de guérison : Max Weber revisité 》, Archives de sciences sociales des religions [En ligne], 113 | janvier-mars 2001, mis en ligne le 19 août 2009,

consulté le 19 avril 2019. URL : http://journals.openedition.org/assr/20196 ; DOI : 10.4000/assr.20196

Ce document a été généré automatiquement le 19 avril 2019.

(C) Archives de sciences sociales des religions 


\title{
Mystagogie et religions de guérison : Max Weber revisité
}

\author{
Régis Dericquebourg
}

1 Max Weber évoque la mystagogie parmi les formes de la domination religieuse. Toutefois, il évoque celle-ci de manière succincte. L'essentiel tient en vingt-cinq lignes dans Économie et Société1. À d'autres endroits, le mystagogue est le plus souvent cité à titre d'exemple, sans plus.

2 D'autre part, le type-idéal du mystagogue n'est pas livré avec la même clarté que celui du prophète, du magicien ou du prêtre. Il comporte de nombreuses lignes de fuites qui le rendent quelque peu difficile à cerner.

3 Dans cet article, nous tenterons de clarifier la notion de mystagogie chez Weber et nous envisagerons sa pertinence pour interpréter l'évolution de certains groupes religieux minoritaires à vocation thérapeutique ${ }^{2}$.

\section{La mystagogie, une définition par touches successives}

Dans sa sociologie de la religion, Max Weber esquisse le type-idéal du mystagogue par une série de démarcations et de rapprochements avec celui du prophète, du magicien et du gourou.

\section{Le mystagogue et le prophète}

Le mystagogue se distingue du prophète mais les deux ne sont pas sans rapports. D'une part, ils ont en commun un charisme; celui du mystagogue est un charisme magique. D'autre part, Max Weber affirme que le type de la prophétie exemplaire se mue très régulièrement en mystagogie dès la seconde génération. Il y a entre eux un lien fonctionnel analogue à la succession du prophète éthique par un administrateur qui pérennise la communauté de disciples. 
6 Le lien successoral entre le prophète exemplaire et le mystagogue est affirmé en ces termes: «Les élèves ou disciples du prophète deviennent alors mystagogues, maîtres, prêtres, directeurs de conscience (ou tout cela à la fois) d'une sociation au service exclusif de fins religieuses : la communauté émotionnelle de laïcs ${ }^{3}$ ». Ici, le mystagogue est lié à des fins religieuses et à l'institutionalisation et non à des fins magiques.

7 L'idée d'une filiation apparaît aussi dans le type-idéal de Max Weber quand ce dernier évoque les dynasties de mystagogues qui se sont «fréquemment développées à partir d'un charisme sacramentel réputé héréditaire " pouvant se ramifier à travers un réseau de disciples grâce à une délégation de pouvoir. L'auteur cite en exemple le gourou de l'Inde ancienne (qui a un sens différent du gourou néo-hindouiste). Mais la transmission du pouvoir annonce le point de dilution de $\mathrm{F}$ idéal-type du mystagogue : celui de la prêtrise héréditaire. Max Weber cite en exemple le prêtre de l'Antiquité grecque qui « préside » - simplement pourrait-t-on dire - aux mystères d'Eleusis.

8 Toutefois, deux caractéristiques distinguent le mystagogue du prophète. D'une part, chez le premier, on constate l'absence de doctrine éthique ou le fait que, si cette dernière existe, elle n'a qu'un «rôle secondaire ». La technique magique semble primer chez le mystagogue. D'autre part, le mystagogue vit de son art à la différence du prophète qui dispense ses biens de salut gratuitement.

\section{Le mystagogue et le magicien}

9 Le lien entre le mystagogue et le magicien est confirmé dans le chapitre que Max Weber consacre à la religiosité asiatique. L'un et l'autre sont classés parmi les sauveurs vivants : "gourous et autres dispensateurs de grâce du même type, que ceux-ci soient plutôt mystagogues ou plutôt magiciens ", "porteurs de charismes » détenus pour la plupart héréditairement. En Asie, à l'exception de la Chine et du Japon où ils ont été réprimés, ils forment une « couche charismatique » qui a « déterminé » la conduite de vie pratique des masses et leur a dispensé un salut magique »; l'abandon au sauveur vivant a constitué le type caractéristique de la piété asiatique ${ }^{4}$.

10 Toutefois, dans les paragraphes qu'il consacre au mystagogue, Weber affirme que ce dernier s'est distingué "graduellement" des magiciens ordinaires par le fait qu'il rassemble autour de lui une communauté de disciples. Cette distinction n'est pertinente que dans ce cas. En effet, des communautés de disciples se forment autour des prophètes mais également autour de personnages non prophétiques: les maîtres spirituels ou les réformateurs par exemple.

11 Si le mystagogue s'est distingué graduellement du magicien, il en est encore proche. Pour Weber, tous deux possèdent aussi un charisme. La formation du magicien consiste précisément à éveiller un don ou à recevoir des techniques purement empiriques ou les deux ${ }^{5}$. Si on poursuit la comparaison sous l'angle de l'éthique, Weber nous apprend qu'il existe une éthique magique. Celle-ci de «l'espèce la plus efficace » est motivée par des normes "purement magiques» dont la violation du point de vue religieux est une abomination ${ }^{6}$. Mais les intérêts visés sont extra-religieux. Par exemple, il ne faut pas irriter les esprits par des conduites qui leur déplaisent pour éviter la maladie; il ne faut pas transgresser le tabou de la séparation des sexes pour préserver la pureté du sang.

12 L'éthique magique se transforme en éthique spécifiquement religieuse quand on passe de la crainte des esprits à la violation des commandements dictés par Dieu. Toutes deux 
peuvent aboutir à une manière de vivre mais dans l'éthique religieuse, on passe au "péché du groupe». Nous revenons ici au prophète. Ce dernier, contrairement au magicien élabore «une centralisation de l'éthique du point de vue de la rédemption religieuse $^{7}$ ». Le contenu de la mission du prophète est le commandement et non point la magie bien que tous deux soient fondés sur un don personnel.

La mystagogie apparait ici comme une transition. Ni magicien ordinaire, ni prophète, le mystagogue peut avoir reçu en héritage de ce dernier la dispensation des biens du salut en usant d'un charisme personnel. À l'opposé peut-il être un personnage qui, au-delà du magicien ordinaire, ébauche à destination d'une communauté de disciples une conception du monde et une éthique religieuse? Peut-il s'orienter vers la gratuité des biens du salut qu'il dispense?

\section{Le mystagogue et le gourou}

La référence que Weber fait au gourou annonce un autre point de dilution du concept de mystagogue.

Le gourou de l'Inde ancienne - on le différencie encore ici du gourou néo-hindouiste - tel qu'il est décrit dans une page précédente ${ }^{8}$ est un simple transmetteur "sur commande » de savoir acquis. Il ne répand pas, de son autorité, un savoir révélé. Toutefois, là encore, s'il est un maître moral, le gourou n'et pas sans lien avec le prophète. Le lien peut s'établir par transitions successives. Les disciples d'un prophète « plein d'une nouvelle sagesse ou de la compréhension renouvelée d'une sagesse ancienne " peuvent devenir les " maîtres moraux » de leurs propres disciples, lesquels s'attachent à lui avec une piété filiale.

Le rapport à un savoir et l'absence de charisme distingue le gourou de l'Inde ancienne du mystagogue.

En somme, les présentations du mystagogue quelque peu buissonnantes que Weber en fait peuvent être synthétisées de la manière suivante.

Weber situe le mystagogue dans l'histoire des mutations du magicien. Il s'agirait d'un magicien qui a réuni des disciples profitant des bienfaits qu'il procure.

Chez lui, des considérations éthiques peuvent exister mais elles passent au second plan. Le mystagogue ne proclame pas une doctrine rédemptrice. En cela, il n'est pas un prophète. Mais si Weber considère que la mystagogie peut être le reliquat ou la suite d'un prophétisme exemplaire, il n'envisage l'évolution de la mystagogie vers le prophétisme ${ }^{9}$ qu'en évoquant la survie d'une lignée de prêtres sacrificateurs par le biais d'une communauté émotionnelle sous des formes liées à la transition de la prophétie mystagogique ou exemplaire. Le cas est spécifique mais intéressant par la question qu'il suscite : peut-on envisager une phase mystagogique dans la construction du prophétisme.

Un mystagogue n'est-il pas conduit parfois à développer une représentation systématisée $\mathrm{du}$ cosmos qui prend un sens par rapport à un Dieu et qui inclut des commandements éthiques voulus par ce Dieu aboutissant à une manière de vivre et à un culte. On peut également se demander si la mystagogie peut comporter des éléments mystiques.

La notion de mystagogie est un point d'ancrage pour l'examen de certaines formes de domination charismatiques transitoires sur une communauté de disciples réunis avant tout pour bénéficier des biens de salut sans recevoir nécessairement une vision du cosmos et les commandements d'un Dieu éthique. Ce qui ne signifie pas que des paroles de sagesse ou des rappels d'une tradition religieuse ne puissent être délivrés. Les disciples 
peuvent payer les bienfaits qu'ils reçoivent. Mais dès que la relation aux disciples devient une simple transmission de savoir ou de savoir-faire, il n'y a plus de mystagogie. Il s'agit d'un professorat.

\section{La mystagogie comme phase intermédiaire de la formation des religions de guérison}

La notion de mystagogie permet-elle d'expliquer la dynamique des groupes religieux minoritaires ? Roy Wallis en a tiré parti dans l'étude de l'Aetherius Society ${ }^{10}$ et dans l'interprétation de la vie du fondateur de la Scientologie ${ }^{11}$. Nous le rejoignons sur ce point et nous pensons que la notion de mystagogie s'applique à la vie des fondateurs de religions de guérison.

Dans la carrière de certains d'entre eux, on trouve une période où le fondateur réunit autour de lui une communauté de disciples qui bénéficie de son charisme de guérison. Des personnes qui ont bénéficié de ses bienfaits et/ou qui tentent d'éveiller des dons de guérison à son contact forment une communauté de disciples qui peuvent le rétribuer et tirer eux-mêmes un revenu de la pratique apprise. À ce stade, ces fondateurs fournissent des explications qui légitiment leur pratique et proposent quelques recommandations éthiques, le plus souvent en réponse aux interrogations des disciples. Toutefois, ils ne livrent pas une vision unitaire et systématique du cosmos et la dispensation des biens du salut magiques passe au premier plan. Selon nous, à ce stade, les fondateurs sont des mystagogues.

On trouve cette traversée mystagogique chez Mary Baker Eddy, Louis Antoine, chez Yvonne Trubert, chez Ron Hubbard respectivement fondateur de la Science chrétienne, de l'Antoinisme, de Invitation à la Vie et de la Scientologie.

\section{Mary Baker Eddy (1821-1910)}

Entre trente et quarante cinq ans, Mary Baker Eddy s'intéresse à divers traitements susceptibles de remédier à sa fragilité et de guérir ses accidents de santé. Elle trouve une première guérison dans le Quimbysme ${ }^{12}$. En 1866, elle fait l'expérience inaugurale de la guérison spirituelle. Paralysée à la suite d'une chute sévère sur le verglas ${ }^{13}$ considérée comme moribonde, Mary Baker Eddy (à l'époque : Mme Patterson) trouve la guérison en méditant sur le récit du paralytique guéri par le Christ (Matthieu : 9, 1-8 ${ }^{14}$ ).

Mary Baker Eddy tente alors de répéter ce fait pour d'autres; elle obtient quelques résultats. Elle fait connaître son expérience autour d'elle et veut en enseigner le modus operandi. Elle trouve son premier disciple dans un compagnon de pension, un cordonnier nommé Hiram Craft qui prête à Mary une écoute intéressée. En la questionnant, Craft, l'oblige à s'expliquer davantage et à construire un système métaphysique à partir de son intuition initiale. La « miraculée de 1866 » commence alors à produire une œuvre fondée sur une interprétation des Écritures. Son élève voulant faire profession de la guérison spirituelle, les Crafts et Mary quittent Lynn pour Taunton où ils ouvrent un cabinet de consultation. L'association est rapidement rompue. Mary Baker Eddy retourne à Lynn en 1870 où elle trouve un nouvel associé, Richard Kennedy. Les rôles sont fixés : elle est le professeur, il est le praticien. Peu à peu, d'autres élèves les rejoignent. Pour subvenir à ses besoins, elle fait payer ses cours. Un cercle de disciples se forme autour d'elle. Toutefois, 
tous ne restent pas fidèles à ses préceptes. Certains versent rapidement dans le mesmérisme en faisant des passes magnétiques à leurs clients pour accélérer la guérison. Mary Baker se sépare d'eux car elle est de plus en plus persuadée que la prière et la méditation sur les Écritures constituent les seuls véritables remèdes.

Avec quelques élèves, elle fonde une société autour de ses idées : l'Association Nationale Scientiste Chrétienne qui se compose à l'origine de sept membres. Suivront quelques années plus tard: L'Association Nationale Scientiste puis L'Eglise du Christ Scientiste de Boston dont elle est le pasteur (1875). Elle crée à Boston en 1881, le Massachusset Metaphysical College. Jusqu'en 1889, date de sa fermeture il a distribué quatre mille diplômes en Science Chrétienne.

Cette période illustre, selon nous la mystagogie comme état intermédiaire. Mary Baker Eddy est une guérisseuse inspirée qui réunit des disciples qui souhaitent bénéficier de son art de guérir et l'apprendre. Son enseignement qui n'est pas gratuit ${ }^{15}$ n'est pas dénué de considérations éthiques mais elles restent au second plan.

Ce n'est que dans une quatrième période que Mary Baker Eddy se consacre totalement à l'annonce d'une voie de salut en accord avec une vision unitaire du cosmos où tout prend un sens par rapport au surnaturel. Elle se fait appeler : Leader et non plus : Teacher. (1888). En 1889, elle dissout les associations fondées antérieurement et effectue un retrait conformément au processus de la construction du charisme prophétique décrite par Roy Wallis ${ }^{16}$. Sa mission est alors soutenue par une communauté de croyants. Elle se consacre à la fondation l'Église de la Science chrétienne. Elle en codifie le fonctionnement, ébauchant déjà, de son vivant, comme l'avait fait Charles Taze Russel, le fondateur des Étudiants de la Bible, un début de routinisation. Elle en confie la gestion à des Conseils.

La séparation entre la phase mystagogique et la phase prophétique n'est pas nette. Pendant la phase mystagogique, elle rédige et publie sa doctrine dans un ouvrage intitulé Science and Health $\left(1875^{17}\right)$. Les éléments de sa cosmologie y sont déjà présents. Elle s'affirme comme prophète lorsqu'elle se consacre totalement et gratuitement à l'annonce de la «Vérité » - Notamment à travers les révisions de son livre de base. La guérison change de plan : elle n'est plus que la démonstration de la "Vérité », - accessible à tous les chrétiens pour peu qu'ils suivent la voie qu'elle indique.

\section{Louis Antoine (1846-1912)}

31 Le fondateur de l'Antoinisme offre un autre exemple. Nous avons décrit ailleurs les trois étapes de sa carrière ${ }^{18}$ : la période spirite (1888-1893), la période d'un culte spontané (1893-1905) et la période prophétique (1905-1912).

Louis Antoine réveille des dons de guérison médiumnique en fréquentant un cercle spirite. Il quitte celui-ci et fonde un groupe kardéciste dont il est la figure de proue. Il manifeste des dons de voyance. En particulier, en atteignant une sorte de transe, il a la vision des organes lésés qu'il va magnétiser. Il rédige un Petit catéchisme spirite et il insiste déjà sur la gratuité des dons. Il délivre des prescriptions simples ("Thé Saint Germain », décoction de fenouil...). Après une condamnation pour exercice illégal de l'art de guérir, il cesse d'interroger les Esprits et de prescrire des posologies. Il en appelle à un Dieu-fluide infini et il obtient des guérisons parmi ceux qui le consultent. Un cercle de disciples se forme autour de lui et le presse de questions sur les fondements de sa pratique. Il y répond ; une sténographe transcrit ses discours. 

livre une vision réincarnationiste du cosmos où la maladie est la conséquence des erreurs commises dans les vies antérieures. En tant qu'épreuve, la maladie procure l'occasion de faire « un travail moral» dont l'exposé devient l'essentiel de la mission. Le traitement spirituel des souffrances occupe peu de place dans son œuvre. Il est la démonstration de la puissance du «travail moral» qui rapproche de Dieu et peut libérer l'homme des incarnations. De surcroît, il privilégie l'évolution morale de l'humanité. disciples adoptent sa tenue vestimentaire (une sorte de lévite noire) et se donnent pour nom : les Antoinistes. Un Conseil de fidèles gère le mouvement antoiniste. Chaque jour à 10 heures, il quitte son logement (où il fait une retraite permanente interrompue par les seuls compagnons autorisés) pour entrer dans le temple adjacent. Il y fait « l'opération générale » (il répand le fluide divin sur l'assemblée gratuitement). Ce culte sera ensuite répété par d'autres. Il est alors prophète d'une religion qui s'est pérennisée. De mystagogue médium des esprits, il passe au stade de «médium de Dieu», prophète. Ses écrits « inspirés » commencent par « Dieu parle ».

S'il n'avait pas élaboré une théodicée qu'il jugeait «inspirée » ou « révélée » et qui fut reconnue par une communauté de disciples, s'il n'avait pas proclamé une éthique de salut individuel et collectif ${ }^{19}$, s'il n'avait pas revendiqué une place dans la lignée prophétique ${ }^{20}$, s'il n'avait pas fait passer la guérison spirituelle au plan de la démonstration gratuite d'une « vérité révélée ", Louis Antoine ne serait resté qu'un thaumaturge local entouré de quelques compagnons et n'aurait pas eu une postérité.

\section{Yvonne Trubert ( 1932-)}

Yvonne Trubert est à l'origine du mouvement Invitation à la Vie. Bretonne venue en apprentissage à Paris puis installée dans la capitale, elle constate qu'elle peut aider les personnes malades ou en détresse par l'écoute et par la prière. Les gens qui s'adressent à elles deviennent si nombreux qu'elle finit par y consacrer tout son temps. Elle découvre l'« harmonisation » qui consiste à masser le corps recouvert d'une étoffe à l'endroit des chakras en priant. Elle découvre ensuite les vibrations qui sont des chants destinés à énergétiser l'homme et le monde. Elle recommande la récitation du rosaire. Elle-même passe de longs moments à prier. La prière, l'harmonisation et les vibrations sont les trois clés de la spititualité Iviste.

37 Yvonne Trubert reçoit et harmonise des personnes souffrantes. Elle trouve parmi elles son premier cercle de disciples à qui elle enseigne l'harmonisation. Ces derniers fondent l'association Invitation à la vie pour la soutenir (1983). Des séminaires de formation à l'harmonisation sont organisés. Les disciples reçoivent à domicile, dans les centres de I.V.I. ou se rendent à l'hôpital. L'harmonisation est considérée comme un acte de charité. Elle n'est pas tarifée mais le patient peut donner une obole pour contribuer à l'œuvre.

L'apport de Yvonne Trubert n'est pas dénué de considérations éthiques. Dans ses conférences, elle rappelle les vertus chrétiennes (en particulier la charité et la compassion) et le modèle de vie évangélique, préconisant en quelque sorte une « imitation de Jésus Christ ». Yvonne Trubert n'a pas acquis un statut de prophétesse. Elle ne se livre pas à une réinterprétation des Écritures, ni à une recomposition doctrinale de type syncrétique comme pourrait le faire penser sa mention des chakras. Elle ne pourrait être prophétesse qu'au sens de l'appellation donnée aux mystiques non-scissionistes du 
Moyen Âge décrites par André Vauchez ${ }^{21}$ qui veulent régénérer (parfois en s'appuyant sur des visions extatiques) le christianisme en admonestant les chrétiens. D'autre part, elle rappelle les Healer-prophets des Églises Indépendantes africaines mais elle n'est pas au service d'une Église et ces derniers ne sont sans doute pas des prophètes au sens wébérien. Ils constituent plutôt une classe de guérisseurs divins qui offrent les mêmes services que les sorciers traditionnels en se référant au Christ au lieu des Esprits ancestraux, contribuant ainsi à l'acculturation des Nouvelles Églises Chrétiennes.

Selon nous, Yvonne Trubert entre dans la catégorie wébérienne du mystagogue. Elle assoie son autorité sur un charisme de guérison et sur la connaissance de techniques de médiation entre Dieu et les hommes qui lui ont été inspirées. Elle pratique et elle enseigne ces dernières mais elle ne livre pas une cosmologie systématisée et ne dicte pas les commandements d'un Dieu éthique. Elle se limite à rappeler des éléments d'une tradition. Maintenant, l'enseignement passe au premier plan. Est-elle en train de sortir de la mystagogie pour évoluer vers un prophétisme? A notre question sur la manière dont elle se perçoit actuellement, elle nous a répondu « plutôt comme un guide spirituel ».

\section{Ron Hubbard (1911-1986)}

La carrière du fondateur de la Scientologie se décompose en deux phases : d'une part, la phase dianétique où Ron Hubbard découvre et diffuse une méthode de guérison et de développement personnel qui repose sur divers éléments puisés dans des théories psychologiques existantes; d'autre part, la phase scientologique proprement dite où après avoir échoué dans sa tentative à imposer la dianétique dans le champ des psychothérapies, il donne à celle-ci une légitimité religieuse en l'inscrivant comme une méthode d'accès à des vies antérieures à l'intérieur d'un système réincarnationniste.

41 Roy Wallis ${ }^{22}$ voit dans la carrière de Ron Hubbard le passage d'un magical healer à un mystagogue puis à un prophète exemplaire. Même si Roy Wallis nous apprend qu'il a été un personnage peu ordinaire, nous sommes réservés à propos du prophétisme (exemplaire) de Ron Hubbard car nous ne sommes pas certains que sa mission ait été gratuite pendant la période scientologique. Nous ne le suivrons pas non plus lorsqu'il considère la phase dianétique comme une phase de magical healer, ou alors il faut considérer tous les psychothérapeutes comme tels. La dianétique est purement technique et ne comporte aucun élément magique. Mais dans la seconde phase, Ron Hubbard appuie son outil de guérison et de développement personnel sur une connaissance supraempirique. Il y ajoute une éthique (« les dynamiques »), un système réincarnationniste, et une voie de la libération (en hâtant la fin des incarnations). Comme psychoreligion proposant la libération totale in fine, la Scientologie fait penser au bouddhisme, mais la phase scientologique de la vie de Ron Hubbard est celle d'un mystagogue, d'un " chercheur spirituel » qui dévoile progressivement ${ }^{23}$ à ses fidèles un enseignement et les instruments d'une libération qui sont tarifés.

Ces prophètes (Mary Baker Eddy, Louis Antoine) et ces mystagogues (Yvonne Trubert, Ron Hubbard) ont des disciples qui perpétuent le charisme de guérison ou les techniques du fondateur en l'inscrivant dans une perspective de salut même si des solliciteurs recourent occasionnellement à leurs service sans entrer dans la voie du salut qu'ils indiquent. Eux-mêmes se conçoivent comme des "pêcheurs d'hommes", parfois des « transmetteurs doctrinaux » rigoureux à la manière d'un maître moral. 


\section{Conclusion}

43 de religions de guérison et de leurs successeurs, que ceux-ci deviennent prophètes ou non.

On trouverait à l'origine de certains mouvements de guérison une phase mystagogique dont les développements peuvent être variés : évolution graduelle vers le prophétisme, mystagogie « avancée », succession mystagogique.

Une autre voie s'ouvre à l'interprétation de certaines pratiques contemporaines: des personnages considérés comme néo-chamans contemporains ou qui se proclament tels sont sans doute plus des mystagogues que des chamans.

\section{NOTES}

1. MaX WEBER, Économie et Société, Paris, Plon, 1971, pp. 470-471.

2. Je remercie Jean Séguy qui, en me relisant, a attiré mon attention sur certaines notions qui y sont discutées.

3. Max WEBER, op.cit., p. 477.

4. Max WEBER, Sociologie de la religion, Paris, Gallimard, 1996, p. 469.

5. Max WEBER, Économie et Société, Paris, Plon, 1971, pp. 450-451.

6. Max WEBER, Économie et Société, Paris, Plon, 1971, p.457.

7. MaX WEBER, Économie et Société. Paris. Plon, 1971, p. 463.

8. MaX WEBER, Économie et société, Paris, Plon, 1971, p. 469.

9. MaX WEBER, Économie et société, Paris, Plon, 1971, p. 477.

10. Roy WALlis, "The Aetherius Society: A case study in the formation of a mystagogie congregation", The Sociological Review, vol. 22/1, 1974, pp. 27-44.

11. Roy WALLIS, The Road to Total Freeedom, Londres, Heinemann, 1976, pp. 248-250.

12. Quimbysme : pratique mesmériste. Quimby avait frictionné la tête de Mary Baker Eddy en évoquant les causes de sa maladie.

13. Un médecin aurait diagnostiqué « une commotion cérébrale et une paraplégie consécutive à une dislocation de l'épine dorsale » (Robert PEEL, Mary Baker Eddy. New York, Holt, Rinehart and Winston, 1972, t. 1, p. 196).

14. Elle a une expérience de lumière qu'elle décrit en ces termes : «La main divine me conduisit dans un monde nouveau de Lumière et de Vie, un nouvel univers - ancien pour Dieu, mais nouveau pour Son petit enfant». Elle a l'impression de comprendre clairement que " tout l'être réel est en Dieu, l'Entendement divin, et que la Vie. la Vérité et l'Amour sont tout puissants et toujours présents $"$.

15. Comme en témoigne cette annonce parue dans le Lynn Seminar Weeky: «Mrs Glover, the wellknown Scientist, will receive applications for one week from ladies and gentlemen who wish to learn how to heal the sick without medecine, and with a success unequaled by anay known method of the present day, at Dr Kennedy office, $n^{\circ} 11$, South Common Street, Lynn, Mass. et ce contrat signé par des élèves: «We, the undersigned, do hereby agree in consideration of 
instruction and manuscripts received from Mrs Mary Baker Glover to pay one hundred dollars in advance and ten per cent, annually on the income that we receive from practising or teaching the science. We also agree to pay her one thousand dollars in case we do note practise or teach the above-mentioned science that she has taught us " in Robert Peel, op.cit., t. 2, pp. 246-247.

16. Roy WALLIS, "The social construction of charisma", Social Compass, XXIX/1, 1982.

17. Tirage à mille exemplaires à compte d'auteur.

18. Régis DERICQUEBOURG, Les Antoinistes, Paris, Brépols, 1993.

19. Résumée dans Les Dix Principes du Père, ed. par les Antoinistes.

20. Louis ANTOINE, Développement de l'enseignement, édité par le mouvement, p. 194 et pp. 200-201.

21. André VAUCHEZ, «Le prophétisme médiéval d'Hildegarde de Bingen à Savonarole, XII-XV siècles ", Politica Hermelica, $\mathrm{n}^{\circ}$ 8. 1994.

22. Roy WALLIS. The Road Total Freedom, Londres, Heinemann, 1976. Pour l'auteur, Ron Hubbard est contraint de pousser ses recherches pour maintenir une domination face aux concurrences dans son domaine.

23. Sur l'établissement d'une autorité sur des personnes par le dévoilement initiatique d'une conception du monde voir Danièle HERVIEU-LÉGER: "Charisma, Utopia and Communal Life. The Case of Neorural Apocalyptique Communes in France”, Social Compass, XXIX/1. 1982, pp. 41-58.

\section{RÉSUMÉS}

Dans cet article, l'auteur tente de reconstituer la définition de la mystagogie chez Max Weber à travers les mentions que ce dernier en fait dans sa sociologie de la religion. En s'intéressant aux applications possibles de cette notion, l'auteur montre qu'elle permet d'interpréter la naissance et l'évolution de certaines religions de guérison occidentales en prenant comme point d'appui la biographie de leurs fondateurs : l'Église de la Science chrétienne, l'Antoinisme, la Scientologie et Invitation à la Vie.

In this paper, the author tries to highlight the definition of the mystagogy as it appears in several paragraphs of the weberian sociology of religion. Applied to the study of cults, the author finds that the notion of mystagogy is useful to understand the emergence and the evolution of four western healing religions from the viewpoint of the biography of their founders: The Christian Science, the Antoinism (a small religious group arised at the end of the $19^{\text {th }}$ century and the beginning of the $20^{\text {th }}$ century), the Church of Scientology and Invitation to life (a French religious movement founded in 1983).

En este artículo, el autor intenta reconstituir la manera en que Max Weber define la mistagogia en varios pasajes de su sociología de la religión. Intersándose en su aplicación para el estudio de diferentes cultos, el autor muestra que est noción permite interpretar el nacimiento y la evolución de cuatro religiones de sonación occidentales desde el punto de vista de la biografía de sus fundadores: la Iglesio de la Ciencia Cristiana, el Antonismo, la Iglesia de Cientología, y Invitación a la vida (un movimiento religioso françés nacido en 1983). 
AUTEUR

RÉGIS DERICQUEBOURG

Groupe de Sociologie des Religions et de la Laïcité - Iresco 